\title{
Antidiabetic potential of turmeric with/without fermented milk enriched with probiotics in diabetic rats
}

\author{
Badkook Maha \\ Department of Food and Nutrition, King AbdulAziz University, Jeddah, Saudi Arabia
}

Email address:

mmbadkook@kau.edu.sa

To cite this article:

Badkook Maha. Antidiabetic Potential of Turmeric with/without Fermented Milk Enriched with Probiotics in Diabetic Rats. American Journal of Biomedical and Life Sciences. Vol. 1, No. 1, 2013, pp. 1-7. doi: 10.11648/j.ajbls.20130101.11

\begin{abstract}
Purpose: To investigate the effect of turmeric supplement (T), fermented milk with Probiotic strain Bifidobacterium lactis DN-173 $010(\mathrm{P})$, a combination of $(\mathrm{T}+\mathrm{P})$ on glycemia, lipidemia, and oxidative status in streptozotocin-induced diabetic rats.Materials and methods: Thirty male albino diabetic rats were fed a normal diet and divided to groups ( $\mathrm{n}=6 /$ group) according to the following treatments for 45 days: Turmeric (T) $(225 \mathrm{mg} / \mathrm{kg} / \mathrm{d})$, fermented milk Probiotics (P) $(0.5 \mathrm{ml} / \mathrm{kg} / \mathrm{d})$, Turmeric-Probiotics combination $(\mathrm{T}+\mathrm{P})(225 \mathrm{mg} / \mathrm{kg} / \mathrm{d}+0.5 \mathrm{ml} / \mathrm{kg} / \mathrm{d})$, Oral hypoglycemic agent (OHA) (0.5 ml of glibenclamide; $450 \mu \mathrm{g} / \mathrm{kg} /$ day), and non-treated (DM). Blood glucose and glycayted hemoglobin were determined after diabetes induction and at the end of experiment. Serum insulin, total cholesterol (TC), LDL-C, HDL-C, TG, malondialdehyde (MDA), and total antioxidant capacity (TOAC) biomarker of oxidative stress were determined at the end of experiment. Results: All treatments resulted in a decrease in glucose and $\mathrm{HbA}_{1} \mathrm{C}$ compared to pretreatment. No difference in insulin concentration was observed. Serum TC was reduced by (T), while LDL-C decreased with $(\mathrm{T})$ and $(\mathrm{T}+\mathrm{P})$ treatments. HDL-C was elevated with all treatments compared to control and (OHA) groups. MDA decreased with all treatments. TOAC was elevated with $(\mathrm{T})$ and $(\mathrm{T}+\mathrm{P})$ but not with $(\mathrm{P})$ treatment. Conclusion: Administration of Turmeric, Probiotics, and $\mathrm{T}+\mathrm{P}$ combination were capable in attenuating hyperglycemia, dyslipidemia and oxidative stress initiated by STZ. However, $(\mathrm{T})$ and $(\mathrm{T}+\mathrm{P})$ exerted a more potent hypolipidemic, and antioxidative effect compared to $(\mathrm{P})$ alone.
\end{abstract}

Keywords: Diabetes, Oxidative Stress, Probiotics, Curcumin, Supplement

\section{Introduction}

Diabetes is associated with diverse chronic cardio and micro-vascular complications as a result of oxidative stress. The increased oxidative stress in type 2 diabetes is a consequence of several abnormalities, including hyperglycemia, insulin resistance, hyperinsulinemia, and dyslipidemia, each of which contributes to mitochondrial superoxide overproduction in endothelial cells of large and small vessels as well as the myocardium.[1]

A large number of studies is in progress to identify natural substances that are effective in reducing those complications associated with type 2 diabetes since insulin and oral hypoglycemic drugs possess undesirable side effects. Spices that are consumed as food adjuncts to enhance sensory quality of foods also possess several medicinal properties and hence find application in the indigenous systems of medicine.[2] Extensive research has shown that the activity of turmeric is due to Curcuminoids, a group of phenolic compounds (mainly diferuloylmethane) isolated from the roots of Curcuma longa (Zingiberaceae), that exhibit a variety of beneficial effects on health which help in preventing certain diseases.[3] Curcumin's potential as a hypoglycemic agent has been studied in animals and humans but with conflicting results.[4,5] Curcumin has been documented to have pronounced hypolipidemic influence in a variety of experimental animal systems. Babu and Srinivasan, (1997) and Srinivasan and Manjunatha, (2007) showed hypolipidemic and antioxidant effects of curcumin $(0.2 \%$, wt/wt) in high-fat $(30 \%)$ fed rats.[6,7] The antioxidative potency of Curcumin was demonstrated in several animal studies.[8-10]

Another functional food that has recently been undergoing much investigation as far as its effect on metabolic diseases is Probiotics. Probiotics are defined as live micro organisms which when administered in adequate amounts confer a health benefit on the host.[11] One type of probiotics, Lactic acid bacteria (LAB), especially 
lactobacilli has been shown to promote beneficial effects in health including anti-cholesterol, antidiabetic, antipathogenic properties, and stimulation of the immune system.[12] Lactobacilli are frequently used in products for human consumption and can be found as probiotics in infant foods, cultured milks, and various pharmaceutical preparations.[12-14] Supplementing the diet with fermented dairy products or lactic acid bacteria containing dairy products has been shown to reduce serum cholesterol levels, as suggested by the results of several human and animal studies.[15] Activia ${ }^{\circledR}$ is fermented milk manufactured with lactic cultures including yoghurt starter cultures (Lactobacillus bulgaricus and Streptococcus thermophilus) and a specific probiotic strain, Bifidobacterium lactis DN-173 010. Studies have recently indicated the role of Probiotics in the modulation of gut microbiota in diabetes conferring a beneficial effect.[16] It is hypothesized that the administration of Probiotics with Turmeric might further enhance the health of diabetic patients when ingested together rather than administration of each functional product by itself.

\section{Materials and Methods}

\subsection{Animals}

Healthy adult male albino Wistar rats $(n=30)$, with a mean weight $(200 \pm 12.34-250 \pm 21.34)$ grams bred in the animal house at King Fahd Medical Research Center (KFMRC), Jeddah, Saudi Arabia were used. All experiments were approved by the (KFMRC) ethical committee. Animals were housed in polycarbonate cages in a room with a $12 \mathrm{~h}$ day-night cycle, temperature of $24 \pm 2{ }^{\circ} \mathrm{C}$, humidity of $50-60 \%$. During the experimental period all groups were maintained on a standard commercial diet (Grain Silos and Flour Mills Organization, KSA) (GSFMO), comprised of wheat starch, casein, soybean oil, cellulose, with mineral and vitamin combinations. The percentage of energy supplied by each macronutrient in the diet was as follows: $66 \%$ carbohydrates, $25 \%$ protein, and $9 \%$ fat. Animals were allowed access to feed and water ad libitum. Fermented milk (Activia ${ }^{\circledR}$, Danone Co.) containing the Probiotic strain Bifidobacterium lactis DN-173 010 was purchased from a local store.

Induction of diabetes was performed by intraperitoneal injection (i.p) of a single dose of Streptozotocin $(65 \mathrm{mg} / \mathrm{kg})$ dissolved in $0.01 \mathrm{M}$ citrate buffer ( $\mathrm{pH} 4.5$ ) immediately before use. After 72 hours, fasting blood glucose was monitored from the tail vein using a glucometer (Bayer ContourTM, USA). Animals having blood glucose levels $<7$ $\mathrm{mmol} / \mathrm{L}$ were excluded from the experiment. Rats were randomly divided into five groups; group (1): DM rats treated with Turmeric supplement (DM+T; $225 \mathrm{mg} / \mathrm{kg} /$ day; $\mathrm{n}=6$ ). Each tablet supplement contains $450 \mathrm{mg}$ curcuminoids + $50 \mathrm{mg}$ turmeric root (Nature's Way Products Inc., Springville Utah, USA), group (2): DM rats treated with an oral daily dose $(0.5 \mathrm{ml} / \mathrm{kg} /$ day Activia fermented milk containing Bifidobacterium lactis DN-173 010 Probiotics $(\mathrm{DM}+\mathrm{P} ; \mathrm{n}=6)$, group (3): DM rats treated with a daily oral dose of $(225 \mathrm{mg} / \mathrm{kg} /$ day Turmeric supplement with 0.5 $\mathrm{ml} / \mathrm{kg} /$ day of Activia fermented milk) $(\mathrm{DM}+\mathrm{T}+\mathrm{P} ; \mathrm{n}=6)$, group (4): DM rats treated with a daily oral dose of $0.5 \mathrm{ml}$ of oral hypoglycemic agent (glibenclamide; $450 \mu \mathrm{g} / \mathrm{kg} /$ day) (DM+OHA, $n=6$ ), group (5): DM control untreated group $(\mathrm{DM}, \mathrm{n}=6)$. Treatments were administered for 45 days after which animals were deprived for 12 hours from food. Before excision animals were anesthetized using diethyl ether. Blood samples were collected from the retro orbital plexus with capillary tubes. Serum was separated by centrifuge at 3000 r.p.m for 15 minutes and aliquots were stored at $-80^{\circ} \mathrm{C}$ until biochemical analysis.

\subsection{Biochemical Analysis}

Fasting blood glucose was determined from the tail vein using a glucometer (Bayer ContourTM, USA) according to the method reported by Tonyushkina \& Nichols (2009).[17] Glycated hemoglobin (HbA1C) was determined in whole blood by fast ion-exchange resin method as described by Nuttall (1998).[18] Serum total cholesterol (TC), high density lipoprotein-Cholesterol (HDL-C), and triglycerides (TG) were assessed using enzymatic colorimetric method as described by Schettler and Nussel (1975), Izawa, et al., (1997), and Fassati and Prencipe (1982) respectively.[19-21] Low density lipoprotein-Cholesterol (LDL-C) was calculated using Friedewald formula (1972).[22] Malondialdehyde (MDA) and total antioxidant capacity (TOAC) were determined colorimetrically according to the method of Ohkawa et al., (1979), and Koracevic et al., (2001) respectively.[23,24] Insulin was determined using enzyme-linked Immunosorbent assay (ELISA) according to the method of Eastham (1985).[25]

\subsection{Statistical Methods}

The statistical package SPSS version 16 (SPSS, Chicago, IL, USA) was used to perform the statistical analysis. The mean and standard error of the mean (SEM) were determined for each biomarker. One- way ANOVA with subsequent post-hoc multiple comparisons tests (Tamhane and LSD) were performed to verify the statistical significance of the studied parameters. Probability values lower than $0.05(\mathrm{p}<0.05)$ were accepted for statistical significance.

\section{Results}

\subsection{Body weight}

At the beginning of the experiment, the range of mean body weights \pm SD was $(200 \pm 12.34-250 \pm 21.34$ gm $)$. All animals gained weight at a similar rate and mean body weights \pm SD were in the range $(287.70 \pm 14.72-339.20 \pm$ $34.27 \mathrm{gm}$ ) at the end of the experiment (Data not shown). No significant difference in body weight gain was observed between groups at the end of intervention. 


\subsection{Effect of Treatments on Glycemic Control}

The ANOVA test showed no significant difference in glucose, glycated hemoglobin ( $\mathrm{HbAlC}$ ), and insulin between groups. The data in table (1) indicates that the administration of all types of treatments: Turmeric, Probiotics, and Turmeric-Probiotics combination resulted in a lower mean \pm SD glucose concentration than the control DM group, even though the difference was not significant. The mean \pm SD concentrations of $\mathrm{HbA1C}$ and insulin of rats given different treatments did not differ significantly from that of OHA and DM control group.
Table 1. Mean serum concentrations of fasting glucose, glycated hemoglobin and insulin at the end of the experiment

\begin{tabular}{llll}
\hline $\begin{array}{l}\text { Treatment } \\
\text { Group }\end{array}$ & FBG $(\mathrm{mmol} / \mathrm{L})$ & $\% \mathrm{HbA}_{1} \mathrm{C}$ & $\begin{array}{l}\text { INSULIN } \\
(\mathrm{ng} / \mathrm{ml})\end{array}$ \\
\hline Turmeric & $12.53 \pm 0.42$ & $7.14 \pm 0.07$ & $2.08 \pm 0.17$ \\
Probiotics & $14.04 \pm 2.45$ & $7.84 \pm 0.17$ & $3.46 \pm 1.15$ \\
T+P & $15.63 \pm 0.49$ & $7.53 \pm 0.11$ & $2.15 \pm 0.28$ \\
OHA & $12.95 \pm 2.53$ & $7.29 \pm 0.17$ & $3.04 \pm 0.88$ \\
DM Control & $19.20 \pm 5.14$ & $7.65 \pm 0.69$ & $2.53 \pm 2.07$ \\
\hline
\end{tabular}

Data represent Mean $\pm S D$. Abbreviations: FBS = fasting blood glucose, $\mathrm{Hb}_{\mathrm{I}} \mathrm{C}=$ Glycated hemoglobin, OHA= Oral hypoglycemic agent .

Table 2 Mean serum concentrations of lipid profile at the end of the experiment

\begin{tabular}{|c|c|c|c|c|}
\hline Treatment Group & CHOL (mg/dl) & HDL-C (mg/dl) & LDL-C (mg/dl) & TG (mg/dl) \\
\hline Turmeric & $91.80 \pm 2.88(.03)^{*}$ & $\begin{array}{c}41.34 \pm 3.61 \\
(.03)^{*},(.02) * *,(.005) * b\end{array}$ & $\begin{array}{c}28.78 \pm 2.58 \\
(\mathrm{p}=0.04) * \mathrm{a},(.004) *\end{array}$ & $172.95 \pm 7.14$ \\
\hline Probiotics & $\begin{array}{c}113.39 \pm \\
13.25\end{array}$ & $\begin{array}{c}42.28 \pm 4.70 \\
(.02)^{*}(.02)^{* *},(.01)^{*} \mathrm{a}\end{array}$ & $\begin{array}{c}41.58 \pm 5.94 \\
* a(p=0.04), * b(p=0.03)\end{array}$ & $120.05 \pm 7.94$ \\
\hline $\begin{array}{c}\mathrm{T}+ \\
\text { Probiotics }\end{array}$ & $\begin{array}{c}97.24 \pm \\
2.17\end{array}$ & $\begin{array}{c}55.42 \pm 3.04 \\
(.01)^{*} \mathrm{a},(.000) *,(0.005) * \mathrm{~b}\end{array}$ & $\begin{array}{c}29.28 \pm 3.00 \\
(.006) *,(p=0.03) * b\end{array}$ & $131.04 \pm 10.86$ \\
\hline OHA & $\begin{array}{c}87.83 \\
\pm 12.51\end{array}$ & $\begin{array}{c}30.50 \pm 5.03 \\
(.02) * *\end{array}$ & $31.67 \pm 11.03$ & $128.33 \pm 36.26$ \\
\hline DM Control & $\begin{array}{c}108.17 \\
\pm 6.67(.03)^{*}\end{array}$ & $\begin{array}{c}31.01 \pm 4.50 \\
*(.02), *(.03), *(.000)\end{array}$ & $\begin{array}{c}47.15 \pm 8.68 \\
*(.004), *(.006)\end{array}$ & $150.00 \pm 18.32$ \\
\hline
\end{tabular}

Data represent Mean $\pm S D . *$ The mean difference is significant at the 0.05 level compared to DM, ** compared to OHA, * a, *b The mean difference between treatments is significant at the 0.05 level. Abbreviations: TC=Total cholesterol, HDL-C=High-density lipoprotein cholesterol, LDL-C= Low-density lipoprotein cholesterol, $T G=$ Triglycerides, OHA= Oral hypoglycemic agent.

\subsection{Effect of Treatments on Lipid Profile}

The ANOVA test indicated a significant difference in total cholesterol (TC), Low-density lipoprotein (LDL-C), and high-density lipoprotein (HDL-C) concentrations between groups at the end of the experiment. As table (2) indicates, the concentration of total cholesterol (TC) decreased significantly in the Turmeric (T) treated group compared to the DM control group ( $\mathrm{p}=0.03$ ). Low-density lipoprotein (LDL-C) concentration was also significantly lower in both the $\mathrm{T}$ and $\mathrm{T}+\mathrm{P}$ groups compared to the $\mathrm{DM}$ group ( $\mathrm{p}=0.004, \mathrm{p}=0.006$ ) respectively. Moreover, LDL-C was significantly lower in both the $\mathrm{T}$, and $\mathrm{T}+\mathrm{P}$ groups than that of the $\mathrm{P}$ group $(\mathrm{p}=0.04, \mathrm{p}=0.03)$ respectively. High-density lipoprotein (HDL-C) concentration was significantly elevated in the $\mathrm{T}, \mathrm{P}$, and $\mathrm{T}+\mathrm{P}$ groups compared to DM control group ( $\mathrm{p}=0.03,0.02, \mathrm{p}<0.001$ respectively). Also, treatment with $\mathrm{T}$, and $\mathrm{P}$ resulted in significantly higher HDL-C levels $(p=0.02)$ compared to the OHA group. High-density lipoprotein was significantly higher in $\mathrm{T}+\mathrm{P}$ group compared to both the Turmeric $(p=0.005)$ and Probiotics $(\mathrm{p}=0.01)$ groups. No significant differences were observed in triglyceride (TG) concentration between treatment groups, or between treatment groups and OHA, or between treatment groups and the DM control group.

\subsection{Effect of Treatments on Oxidative Stress}

The ANOVA test indicated a significant difference in the concentrations of both biomarkers of oxidative stress compared to DM control group at the end of the experiment.

Malondialdehyde (MDA) concentration was significantly reduced in all treatment groups $(\mathrm{T}, \mathrm{p}=0.04)(\mathrm{P}, \mathrm{p}=0.033)$ and $(\mathrm{T}+\mathrm{P}, \mathrm{p}=0.04)$ compared to the DM control group (figure 1$)$. On the other hand, the total antioxidant capacity (TOAC) was significantly elevated in the $\mathrm{T}$ and $\mathrm{T}+\mathrm{P}$ groups $(\mathrm{p}<$ 0.001) compared to OHA and DM control group (figure 2). However, TOAC concentration was lower in the $\mathrm{P}$ group compared to the $\mathrm{T}$ group $(\mathrm{p}=0.02)$.

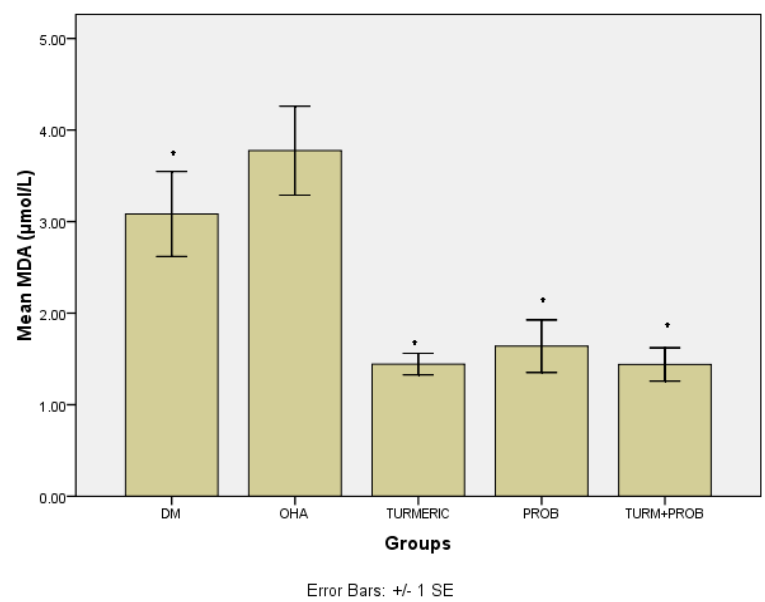

Figure 1 Effect of treatments on lipid peroxidation $(M D A)$ * the mean difference is significant at the 0.05 level compared to DM. Abbreviations: DM=Diabetes Mellitus control, Prob $=$ probiotic, TURM $+P R O B=$ Turmeric + probiotic, OHA = Oral hypoglycemic agent. 


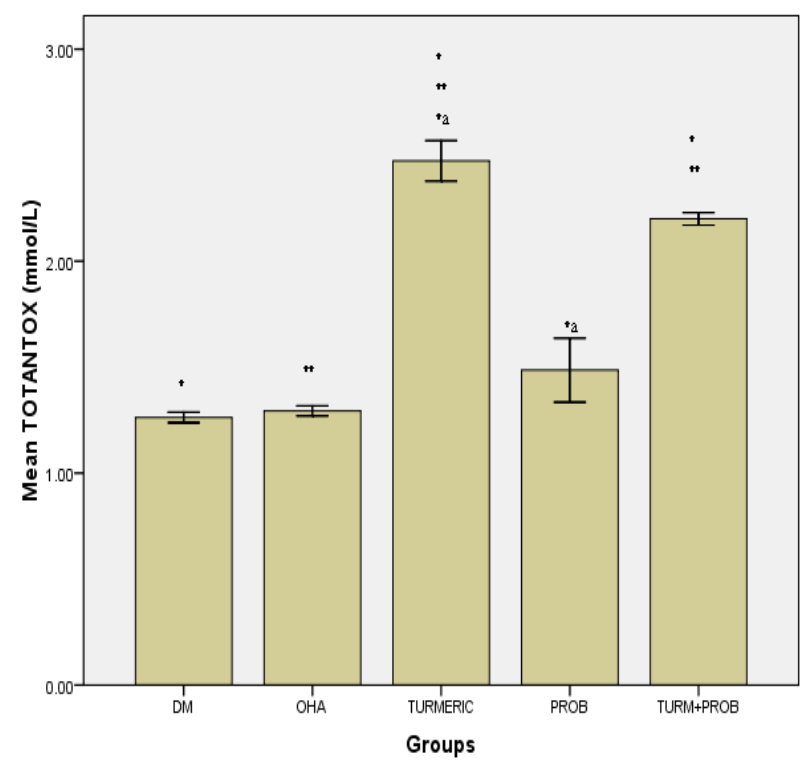

Figure 2 Effect of treatments on oxidative stress (TOAC) * the mean difference is significant at the 0.05 level compared to DM, ** the mean difference is significant at the 0.05 level compared to $O H A, * a$, the mean difference between treatments is significant at the 0.05 level. Abbreviations: $D M=$ Diabetes Mellitus control, Prob $=$ probiotic, $T U R M+P R O B=$ Turmeric + probiotic, $O H A=$ Oral hypoglycemic agent.

\section{Discussion}

Management of diabetes without any side effects is still a challenge to the medical system. Studies in animals and short-term human studies have suggested that Curcumin, the polyphenolic concentrated compound in turmeric, improved glycemic, hypolipidemic and antioxidative effects in type 2 diabetes. [26, 27]

In this study, the administration of $225 \mathrm{mg} / \mathrm{kg} / \mathrm{day}$ of Turmeric supplement, Probiotics, and the Turmeric-Probiotics combination to STZ-diabetic rats for 45 days, improved the glycemic state as indicated by the reduction in fasting blood glucose and $\mathrm{HbA} 1 \mathrm{C}$ at the end of the experiment compared to pre-treatment levels. Moreover, the levels of glucose and $\mathrm{HbA} 1 \mathrm{C}$ were lower in the $\mathrm{T}$ and $\mathrm{P}$ treatment groups compared to the OHA and the DM control groups, at the end of the experiment, but not in the $\mathrm{T}+\mathrm{P}$ group. Although this reduction was not significant, however, it suggests a beneficial glycemic regulatory effect exerted by such treatments. Fujiwara et al., (2008) studied Curcumin effect on hepatic glucose production, and reported that the anti-diabetic effects of Curcumin are partly due to a reduction in hepatic glucose production.[28] Similarly, the study of Pari and Murugan, (2007) indicated a significant reduction in blood glucose in STZ -diabetic rats fed 80 $\mathrm{mg} / \mathrm{kg}$ tetrahydrocurcuminoids.[26] Comparing two doses of Turmeric supplementation $(30 \mathrm{mg} / \mathrm{kg}, \quad 300 \mathrm{mg} / \mathrm{kg})$ Rungseesantivanon et al., (2010) showed that $(30 \mathrm{mg} / \mathrm{kg})$ resulted in significantly lower levels of $\mathrm{HbA} 1 \mathrm{C}$ compared to DM group, while the higher dose $(300 \mathrm{mg} / \mathrm{kg})$ resulted in only a $32 \%$ decrease in plasma glucose.[5] Shehzad et al., (2011) pointed out that the interactions of curcumin with several signal transduction pathways reverse insulin resistance, hyperglycemia, hyperlipidemia.[29]

Treatment with probiotics has been suggested to have a potent effect in type 2 diabetes by improving the endogenous flora as previous studies suggest that modulating gut microbiota could be beneficial for improving glycemic control.[16] Al-Salami et al., (2008) have shown that probiotic treatment of diabetic rats given gliclazide, increased its bioavailability and lowered blood glucose levels by insulin-independent mechanisms, suggesting that the administration of probiotics may be beneficial as adjunct therapy in the treatment of diabetes.[30] Although the administration of Turmeric $(\mathrm{T})$ and turmeric-probiotics combination $(\mathrm{T}+\mathrm{P})$ were capable of reducing serum glucose levels, it is apparent that Turmeric when given alone was more effective $(p<0.001)$ in attenuating serum glucose than $\mathrm{T}+\mathrm{P}$ combination. The results might have been due to the low dose of probiotics administered in this study, since $\mathrm{T}+\mathrm{P}$ treatment was capable of suppressing the rise in glucose and $\mathrm{HbA} 1 \mathrm{C}$ levels caused by induction of diabetes before intervention.

The non-significant difference observed in serum insulin levels between treatment groups and the OHA and DM control groups might indicate that either the dose or the duration of treatment was not sufficient to improve insulin secretion, or that STZ injection resulted in excessive and irreversible destruction of the beta cells of the pancreas. Pari and Murugan, (2007) observed a significant increase in plasma insulin in diabetic rats given tetrahydrocurcuminoids (THC) but not in those given Turmeric, indicating that THC possess an anti-diabetic effect.[26]

There is sufficient evidence that Curcumin induces a hypolipidemic effect in type 2 diabetes primarily in animal models. Early studies on Curcumin have shown a decrease in total plasma cholesterol in mice, rats, and rabbits.[31-33]

Results of this study indicate that treatment with $225 \mathrm{mg} / \mathrm{kg} /$ day of Turmeric, significantly lowered TC and LDL-C compared to DM control group. Although the TC level of the $\mathrm{P}$ or $\mathrm{T}+\mathrm{P}$ treatments were not different to that of $\mathrm{DM}$ or $\mathrm{OHA}$ groups, however, $\mathrm{T}+\mathrm{P}$ treatment was capable of reducing LDL-C level significantly compared to DM group. This suggests that both $\mathrm{T}$ and $\mathrm{T}+\mathrm{P}$ treatments exerted a hypolipidemic effect, while treatment with Probiotics alone did not. On the other hand, all treatments did not cause significant change in TG levels compared to DM control or OHA groups. These results are partially consistent with the study of Jang, (2008) who reported a significantly lower levels of TC, and TG in high-fat fed hamsters treated with Curcumin (0.05-g/100-g diet), while HDL-C were elevated.[10] Also, Kempaiah and Srinivasan (2004) showed that rats fed HF diet (30\%) with Curcumin $(0.2 \%)$ for eight weeks resulted in a hypotriglyceridemic effect.[9] The partial inconsistency in the findings of this study compared to the two mentioned studies might be interpreted by the difference in the physiological state of the animals, and the high-fat diet administered in those previous studies. 
The significant elevation in HDL-C level observed in all treatment groups compared to the DM and OHA groups indicates a beneficial effect exerted by these treatments on the dyslipidemia associated with diabetes.

One beneficial effect that has been suggested from human consumption of the lactobacillus (LAB) probiotics is a reduction in serum cholesterol levels, as suggested by the results of several human and animal studies. [15] This effect can partially be ascribed to an enzymatic deconjugation of bile acids. Cholesterol, being a precursor of bile acids, converts its molecules to bile acids replacing those lost during excretion leading to a reduction in serum cholesterol .[34]

Unexpectedly, in this study the type of probiotics administered produced a non-significant effect in TC, LDL-C, and TG compared to the DM control group. This could be related to the type of probiotics strain used, or that the dose was not sufficient to exert a hypolipidemic effect. However, the rise in HDL-C accompanying the probiotics treatment could outweigh the null effect perceived with the other serum lipid components. Moreover, the lower level observed in the $\mathrm{T}$ group compared to the $\mathrm{P}$ group indicates that ingestion of Turmeric outperforms probiotics in regulating TC level. In accordance with our findings, Sadrzadeh-Yeganeh et al., (2010) reported no difference in TG and LDL-C, and a decrease in cholesterol in subjects who consumed both conventional and probiotic yoghurt groups compared with the control group, and an increase in HDL-cholesterol in the probiotic yoghurt group compared with the control group.[35] Thus, supplementation of different strains of probiotics indicate controversial results which might be related to the type of probiotics strain and/or the dose used.

The induction of diabetes using Streptozotocin has been shown to cause increased generation of reactive oxygen species, and the majority of the beta cell damage is attributed to the extensive oxidative stress caused by STZ-induced depletion of free radical scavenging potential. [36] There is sufficient evidence that treatment with Curcumin ameliorates the oxidative stress induced by STZ. Several studies have shown increased lipid peroxidation in clinical and experimental diabetes. [37,8]

In this study, significant differences in malondialdehyde level was observed in the STZ-diabetic groups treated with either $\mathrm{T}, \mathrm{P}$, or $\mathrm{T}+\mathrm{P}$ combination, compared to the $\mathrm{DM}$ control group $(\mathrm{p} \leq 0.05)$. This indicates that all types of treatments were capable of reducing lipid peroxidation encountered in diabetes. In accordance with those results, Murugan and Pari, (2006) observed significant increases in antioxidant enzymes and lower Thiobarbituric acid reactive substances (TBARS) in STZ-diabetic rats fed THC at 80 $\mathrm{mg} / \mathrm{kg}$ body weight of for 45 days.[38] Similarly, Kempaiah and Srinivasan, (2004) reported that addition of Turmeric $(0.2 \%)$ to HF $(30 \%)$ diets rats fed 8weeks decreased lipid peroxidation and was effective in reducing oxidant stress.[9] In high-fat fed hamsters, treatment with Turmeric (0.05-g/100-g diet) significantly lowered the lipid peroxide levels in the erythrocyte and liver.[9] Al-Suhaimi et al., (2011) explained that turmeric might exert its actions as a potent inhibitor of reactive oxygen species (ROS)-generating enzymes, which play an essential role in inflammation and progression of diseases.[39]

In the present study, rats treated with either Turmeric, or $\mathrm{T}+\mathrm{P}$ combination showed a significant increase in total antioxidant capacity (TOAC) compared to DM and OHA groups implying an anti-oxidative benefit of these treatments. Moreover, the $\mathrm{T}$ group showed significantly higher TOAC level than $\mathrm{P}$ alone. This might indicate that, for Probiotics to be able to promote an anti-oxidative benefit it has to be ingested in combination with Turmeric which itself has an established evidence of anti-oxidative potential. Despite that, few studies have been conducted regarding the effect of Probiotics on oxidative stress, with controversial results reported in human studies. Whereas Fabian and Elmadfa, (2007) showed a significant decrease in the average TOAC and increased MDA values with the intake of probiotic (Lactobacillus casei) compared to conventional yoghurt,[40] another study on type 2 diabetic patients, indicated that consumption of probiotic yogurt compared to conventional yogurt for 6 weeks, increased antioxidant enzyme activities and total antioxidant status in the Probiotics compared with the control group.[35] These controversies imply the need for further studies on the anti-oxidative properties of different strains of probiotics.

\section{Conclusion}

The effect of Turmeric, Probiotics, and their combination on glucose homeostasis, dyslipidemia, and oxidative stress in STZ-diabetic rats showed a significant reduction in fasting blood glucose and HbA1c levels after 45 days of treatment indicating a beneficial effect in controlling hyperglycemia. Also, the reduction in TC and LDL-C caused by $\mathrm{T}$ and $\mathrm{T}+\mathrm{P}$ supplements, and the elevation of HDL-C exerted by all treatments demonstrates their capability in ameliorating dyslipidemia. The significant reduction in MDA after $\mathrm{T}$ and $\mathrm{T}+\mathrm{P}$ administration implies that both types of supplementations were effective in diminishing the rate of lipid peroxidation encountered in diabetes. In addition, the elevation of TOAC observed with all three types of supplementations further indicates their anti-oxidative potential, although $\mathrm{T}$ and $\mathrm{T}+\mathrm{P}$ treatments demonstrated a more potent effect than Probiotics alone.

Overall, while the three types of treatments exerted a hypoglycemic effect, treatments with $\mathrm{T}$, and $\mathrm{T}+\mathrm{P}$ only were capable of attenuating dyslipidemia. On the other hand, the reduction of MDA and elevation of TOAC levels observed by the administration of all types of supplements for 45 days implies that all treatments possess anti-oxidative property which might enhance diabetic patients' health. Further studies are required utilizing different doses and strains of Probiotics and probiotics in combination with functional products to examine their effects on the metabolic regulation of diabetes. 


\section{Acknowledgments}

The author is grateful to Ms. Sonham Yaghmour for performing some biochemical tests. The author got financial support as a grant to conduct this research from King AbdulAziz University.

\section{References}

[1] Folli F, Corradi D, Fanti P, Davalli A, Paez A, Giaccari A, Perego C, Muscogiuri $G$ The role of oxidative stress in the pathogenesis of type 2 diabetes mellitus micro- and macrovascular complications: avenues for a mechanistic-based therapeutic approach. Curr Diabetes Rev. 2011; 7(5): 313-24.

[2] Kempaiah RK, Srinivasan K. Beneficial influence of dietary curcumin, capsaicin and garlic on erythrocyte integrity in high-fat fed rats. J Nutr Biochem. 2006; 17(7) 471- 478.

[3] Joe B, Vijaykumar M, Lokesh BR. Biological properties of curcumin-cellular and molecular mechanisms of action. Crit Rev Food Sci Nutr 2004; 44(2): 97-111.

[4] Pari L, Murugan P. Effect of tetrahydrocurcumin on blood glucose, plasma insulin and hepatic key enzymes in streptozotocin induced diabetic rats. J Basic Clin Physiol Pharmacol 2005; 16: 257-274.

[5] Rungseesantivanon S, Thenchaisri N, Ruangvejvorachai P, Patumraj S. Curcumin supplementation could improve diabetes-induced endothelial dysfunction associated with decreased vascular superoxide production and PKC inhibition. BMC Complement Altern Med. 2010; 10: 57-62.

[6] Babu SP, Srinivasan K. Hypolipidemic action of curcumin, the active principle of turmeric Curcuma longa in streptozotocin induced diabetic rats. $\mathrm{Mol}$ and Cellular Biochem. 1997; 166: 169-175.

[7] Srinivasan K, Manjunatha H. Hypolipidemic and antioxidant effects of curcumin and capsaicin in high-fat-fed rats. Can J Physiol Pharmacol 2007; 85:588-96.

[8] Eshrat HM, Hussain A. Hypoglycemic, hypolipidemic and antioxidant properties of combination of Curcumin from CURCUMA LONGA and partially purified product from ABROMA AUGUSTA, in Streptozotocin induced diabetes. Indian J Clin Biochem. 2002; 17 (2): 33-43.

[9] Kempaiah RK, Srinivasan K. Influence of dietary curcumin, capsaicin and garlic on the antioxidant status of red blood cells and the liver in high-fat-fed rats. Ann Nutr Metab. 2004; 48(5): 314-20.

[10] Jang E-M, Choi M-S, Jung UJ, Kim M-J, Kim H-J, Jeon $\mathrm{S}-\mathrm{M}$, et al. Beneficial effects of curcumin on hyperlipidemia and insulin resistance in high-fat-fed hamsters. Metab Clin and Exp. 2008; 57: 1576-1583.

[11] Iannitti T, Palmieri B.Therapeutical use of probiotic formulations in clinical practice.Clin Nutr. 2010; 29(6):701-25.

[12] Nagpal R, Yadav H, Puniya AK, Singh K, Jain S, Marotta F. Potential of probiotics and prebiotics for synbiotic functional dairy foods. Int. J Probiotics and Prebiotics 2007; $2: 75-84$.
[13] Sanders TAB. Food production and food safety. BMJ 1999; 318(7199):1689-1693.

[14] Nagpal R, Behare PV, Kumar M, et al. Milk, milk products and disease free health: an updated overview. Crit Rev Food Sci and Nutr 2011; 52(4):1549-52.

[15] Pereira DIA, Gibson GR. Effects of consumption of probiotics and prebiotics on serum lipid levels in humans. Crit Rev Biochem and Mol Biol 2002; 37(4):259-281.

[16] Cani PD, Delzenne NM. The role of the gut microbiota in energy metabolism and metabolic disease. Curr Pharm Des. 2009; 15(13): 1546-58.

[17] Tonyushkina K, Nichols JH. Glucose meters: a review of technical challenges to obtaining accurate results, J Diabetes Sci Technol. 2009; 3: 971-980.

[18] Nuttall FQ (1998) Comparison of percent total GHb with percent $\mathrm{HbA} 1 \mathrm{c}$ in people with and without known diabetes. Diabetes Care 1998; 21(9):1475-80.

[19] SchettlerG, Nussel E. Enzymatic calorimetric determination of high density lipoprotein cholesterol by CHOD-PAP method, Arb. Med Soz. Med Prov Med. 1975;10:25.

[20] Izawa S, Okada M, Matsui H, Horita Y. A new direct method for measuring HDL cholesterol which does not produce any biased values, J. Med. Pharm. Sci 1997; $137: 1385-1388$

[21] Fassati P, Prencipe L. Triglyceride enzymatic colorimetric method. Clin Chem 1982; 28: 2077.

[22] Friedewald, W.T., Levy, R.I., Fredrickson, D.S. Estimation of the concentration of low-density lipoprotein cholesterol in plasma without use of preparative ultracentrifuge. Clin Chem 1972; 18 (6): 499- 502.

[23] Ohkawa, H., Ohishi, N., \& Yagi, K. Assay for lipid peroxides in animal tissue by thiobarbituric acid reaction. Analytical Biochemistry 1979; 95(2): 351-58.

[24] Koracevic, D; Koracevic, G; Djordjevic, V; Andrejevic, S; Cosic, V. Method for the measurement of antioxidant activity in human fluids. J Clin Pathol. 2001; 54; 5 : 356-61.

[25] Eastham RD. Biochemical values in clinical medicine. $7^{\text {th }}$ ed. Bristol, England John Wright \& Sons Ltd. (1985)

[26] Pari L, Murugan P. Antihyperlipidemic effect of curcumin and tetrahydrocurcumin in experimental type 2 diabetic rats. Ren Fail. 2007; 29(7): 881-9.

[27] Menon VP, Sudheer AR. Antioxidant and anti-inflammatory properties of curcumin. Adv Exp Med Biol. 2007;595:105-25.

[28] Fujiwara H, Hosokawa M, Zhou X, Fujimoto S, Fukuda K, Toyoda $\mathrm{K}$, et al. Curcumin inhibits glucose production in isolated mice Hepatocytes. Diabetes Res and Clin Pract. 2008; 80:185-91

[29] Shehzad A, Ha T, Subhan F, Lee YS. New mechanisms and the anti-inflammatory role of curcumin in obesity and obesity-related metabolic diseases. Eur J Nutr. 2011; 50(3):151-61.

[30] Al-Salami H, Butt G, Fawcett JP, Tucker IG, Golocorbin-Kon S, Mikov M. Probiotic treatment reduces blood glucose levels and increases systemic absorption of 
gliclazide in diabetic rats. Eur J Drug Metab Pharmacokinet. 2008; 33(2):101-6.

[31] Soudamini KK, Unnikrishnan MC, Soni KB, Kuttan R. Inhibition of lipid

[32] peroxidation and cholesterol levels in mice by curcumin. Indian J Physiol Pharmacol. 1992; 36:239-43.

[33] Arafa HM. Curcumin attenuates diet-induced hypercholesterolemia in rats. Med Sci Monit. 2005; 11:BR228-34.

[34] Ramirez-Tortosa MC, Mesa MD, Aguilera MC, Quiles JL, Baro L. Oral administration of a turmeric extract inhibits LDL oxidation and has hypocholesterolemic effects in rabbits with experimental atherosclerosis. Atherosclerosis 1999; 147:371-8.

[35] Usman HA. Bile tolerance, taurocholate deconjugation, and binding of cholesterol by Lactobacillus gasseri strains. J Dairy Sci. 1999; 82(2):243-248.

[36] Sadrzadeh-Yeganeh H, Elmadfa I, Djazayery A, Jalali M, Heshmat R, Chamary M. The effects of probiotic and conventional yoghurt on lipid profile in women. $\mathrm{Br} \mathrm{J}$ Nutr. 2010; 103(12):1778-83.
[37] Meghana K, Sanjeev G, Ramesh B. Curcumin prevents streptozotocin-induced islet damage by scavenging free radicals: A prophylactic and protective role. European J Pharm. 2007; 577: 183-191.

[38] Sundaram, R.K., Bhaskar, A., Vijayalingam, S., Viswanatthan, M., Moha, R., Shanmugasundaram, K.R. Antioxidant status and lipid peroxidation in type II diabetes mellitus with and without complications. Clin Sci. 1996; 90, 255-60.

[39] Murugan P, Pari L. Antioxidant effect of tetrahydrocurcumin in streptozotocin-nicotinamide induced diabetic rats. Life Sciences 2006; 79:1720-1728.

[40] Al-Suhaimi EA, Al-Riziza NA, Al-Essa RA. Physiological and therapeutical roles of ginger and turmeric on endocrine functions. Am J Chin Med. 2011; 39(2):215-31.

[41] Fabian E, Elmadfa I. The effect of daily consumption of probiotic and conventional yoghurt on oxidant and anti-oxidant parameters in plasma of young healthy women. Int J Vitam Nutr Res. 2007; 77(2):79-88. 